\title{
The Effects of Pruning on Drag and Bending Moment of Shade Trees
}

\author{
Michael Pavlis, Brian Kane, J. Roger Harris, and John R. Seiler
}

\begin{abstract}
Arborists assume that pruning can help reduce the risk of tree failure by reducing the pressure exerted on trunks by wind (drag-induced bending moment), but there are few studies that quantify this effect. We simulated wind by driving trees in the back of a pickup truck from 0 to $24.5 \mathrm{~m} / \mathrm{s}(0$ to $55 \mathrm{mph}$ ) and measured drag-induced bending moment as well as tree morphometric data for Freeman maple (Acer $\times$ freemanii), swamp white oak (Quercus bicolor Willd.), and shingle oak (Quercus imbricaria Michx.). Measurements were taken before and after application of one of three American National Standards Institute A300 pruning types (raising, reduction pruning, thinning). Reduction of drag-induced bending moment differed by pruning type, largely in accordance with the mass of foliage and twigs removed. The effectiveness of pruning types was also species-dependent because crown architecture affected how much mass each pruning type removed. In general, per unit of mass removed, reduction pruning more effectively reduced the drag-induced bending moment than thinning or raising. Reduction pruning reduced the center of pressure height and, presumably, increased crown porosity after pruning. Prediction of the reduction of drag-induced bending moment was not reliable based on reduction in crown area after pruning. We discuss the practical applications of our findings.
\end{abstract}

Key Words. Bending moment; drag; pruning.

Arborists commonly prune trees for a variety of reasons, including risk reduction, maintaining tree health, and aesthetics. Historically, in the United States, trees were topped and lion-tailed to reduce the risk of tree failure; topping was expected to reduce windthrow by reducing tree height and therefore the leverage the wind could exert on it. Lion's-tailing was expected to facilitate wind passage through the crown by making it more porous. These pruning practices, however, are no longer recommended (Lilly 2001) nor were their presumed benefits ever rigorously quantified. Instead, for arborists in the United States, the A300 Standard (American National Standards Institute [ANSI] 2001) describes four conventional pruning types and requires arborists who adhere to the standards to develop objectives before prescribing a particular pruning type(s). Pruning types and prescriptions have been developed mostly without the benefit of empiric tests of shade trees. This is especially true with respect to the effect of pruning on drag reduction, and, consequently, reducing the risk of tree or branch failure. Some studies conducted in wind tunnels have investigated the effect of pruning on drag of conifers (Fraser 1962; Mayhead et al. 1975; Rudnicki et al. 2004) and deciduous trees (Vollsinger et al. 2005), but interpretation of results is limited because few replications were used (Fraser 1962; Mayhead et al. 1975) and trees were small (less than $2 \mathrm{~m}$ [6.6 ft] tall) (Rudnicki et al. 2004; Vollsinger et al. 2005). Smiley and Kane (2006) examined the effect of pruning on drag reduction, but the study included only a single species (red maple [Acer rubrum L.]) and three wind speeds.

Recent efforts in Europe to assess tree risk using a pull test have led to the development of prescriptive pruning to reduce drag consistent with risk reduction (Wessolly 1995). Such efforts are a useful start to address risk reduction issues more quantitatively, but the pruning prescription, which amounts to a series of crown reductions, is based on a theoretical reduction in drag (D) from the classic equation,

$$
\mathrm{D}=0.5 * \rho^{*} \mathrm{~A} * \mathrm{C}_{\mathrm{D}} * \mathrm{U}^{2}
$$

where $\rho$ is air density, $A$ is crown frontal area, $C_{D}$ is the drag coefficient, and $\mathrm{U}$ is wind speed. The fundamental premise is that crown reduction reduces drag consistent with crown area reduction and lowers the center of pressure height of the crown. Crown reduction thus has the effect of reducing bending moment, which is the product of drag and center of pressure height. Crown reduction also reduces the wind speed to which the tree is exposed because wind speed increases logarithmically with distance above the ground (Davenport 1968). Although Wessolly (1995) did not test trees to determine whether the predicted reduction in drag and bending moment was realized, Smiley and Kane's (2006) results suggest that per unit of crown mass removed, reduction pruning more effectively reduced bending moment than thinning and lion's-tailing.

Many questions remain, however, because drag coefficients among species differ widely (Mayhead et al. 1975; Rudnicki et al. 2004; Vollsinger et al. 2005; Kane and Smiley 2006). This suggests that species may respond differently to different pruning types. The objectives of this research were to investigate:

1. The effect of pruning on drag and bending moment of shade trees;

2. The effect of wind speed on drag and bending moment of shade trees;

3. Which tree morphometric data best predict the effect of pruning on drag and bending moment of shade trees; and

4. Whether species influenced the effect(s) of pruning and wind speed on drag and bending moment.

\section{METHODOLOGY}

\section{Trees}

We tested three shade tree species, Freeman maple (Acer $\times$ freemanii) —a cross between red and silver (Acer saccharinum 
L.) maples, swamp white oak (Quercus bicolor Willd.), and shingle oak (Quercus imbricaria Michx.) (Table 1; Figure 1). Freeman maple has been planted as a street tree across the eastern United States. Swamp white oak has been suggested as a good street tree because of its tolerance of poor soil (Dirr 1997). Shingle oak has been increasingly requested as a street tree according to urban foresters in Ohio (D'Amato et al. 2002). All trees were grown at the Urban Horticulture Research Center in Blacksburg, Virginia, U.S. (USDA hardiness zone 7a) and testing was conducted during July and August 2005.

We cut trees just above the root flare and fastened the entire tree above the cut in a steel sled mounted in the rear of a pickup truck (Figure 2). The truck was then driven on a straight, nearly level course from 0 to $24.5 \mathrm{~m} / \mathrm{s}$ (0 to $55 \mathrm{mph}$ ). Although wind flow was not laminar like in a wind tunnel, this method enabled us to test larger trees. To improve experimental rigor, we refined Smiley and Kane's (2006) method as noted subsequently.

\section{Wind Measurements}

We measured wind speeds with two three-cup anemometers, one attached to either side of the cab of the pickup truck. Anemometers on the left and right sides of the truck were attached, respectively, 0.8 and $2.2 \mathrm{~m}(2.6$ and $7.3 \mathrm{ft})$ above the cab (Figure 2 ). Wind speed data were collected at $1 \mathrm{~Hz}$ with Sensormetrics ${ }^{\circledR}$ (Southbridge, MA) software. The anemometers were calibrated with the truck's speedometer on each day of testing after the truck's speedometer was calibrated with a radar gun before any testing. To reduce further the error resulting from ambient wind, we tested each tree twice, driving it in opposite directions on the test course, and we did not conduct tests when ambient wind speed exceeded $1.4 \mathrm{~m} / \mathrm{s}$. Because wind speeds measured with each anemometer did not differ beyond their inherent error $(0.5$ $\mathrm{m} / \mathrm{s}$ ), we averaged their values.

\section{Load Measurements}

We measured wind loads with a 9,800 N capacity Dillon EDxtreme dynamometer (Weigh-Tronix, Fairmont, MN; accurate to $9.8 \mathrm{~N}$ ) and collected data at $2 \mathrm{~Hz}$ using Wedgelink ${ }^{\circledR}$ software (MicroRidge Systems, Inc., Sunriver, OR). The dynamometer was attached to an anchor point on the sled by a steel cable and to the tree by a polyester webbing sling. We used moment equilibrium to convert measured loads (L) into actual drag (D),

$$
\mathrm{D}=\mathrm{L} * 0.76 / \mathrm{CPH}
$$

where $\mathrm{CPH}$ is the center of pressure height, explained subsequently (for details of this procedure, see Kane and Smiley [2006]).

Because wind speeds were recorded one-half as frequently as load measurements, wind speeds at subsequent $1 \mathrm{sec}$ intervals

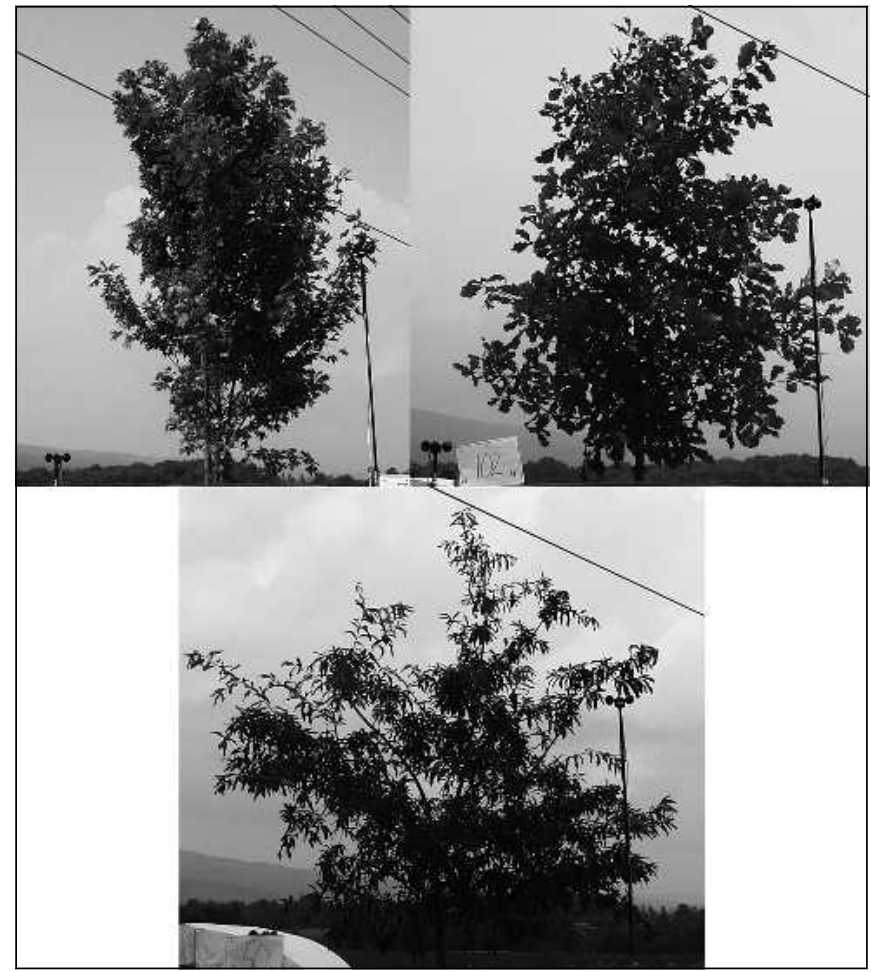

Figure 1. Typical forms for, clockwise from top left, Freeman maple, swamp white oak, and shingle oak tested in the current study. The distance from the bottom of the image to the anemometer on the right side of each image represents $\mathbf{2 . 2}$ $\mathrm{m}(7.3 \mathrm{ft})$.

were averaged to create values for each half-second interval. For wind speeds between 13.4 and $24.5 \mathrm{~m} / \mathrm{s}$ (30 and $55 \mathrm{mph}$ ), we plotted drag versus wind speed from both test runs of each tree and fit a straight line to the data. From the line, we predicted drag at 5 wind speeds $(13.4,15.6,17.9,20.1,22.4 \mathrm{~m} / \mathrm{s}$ [30, 35, 40, 45, $50 \mathrm{mph}])$ for each tree. If the coefficient of determination for the line was less than 0.90 , we plotted each test run separately. In five cases (two Freeman maples, two swamp white oaks, and one shingle oak), doing so did not improve the coefficient of determination, so those trees were removed from the analysis.

Because we recorded instantaneous wind speeds, measured loads included a component of force resulting from vehicular acceleration. We determined vehicular acceleration by plotting wind speed against time for each tree; the slope of the best-fit line for the plot is the acceleration. Acceleration was nonlinear at speeds up to approximately $11.0 \mathrm{~m} / \mathrm{s}$ but consistently linear from 13.4 to $24.5 \mathrm{~m} / \mathrm{s}$ (30 and $55 \mathrm{mph}$ ), so we only plotted speeds in

Table 1. Means (standard deviation in parentheses) for tree morphometric measures describing each species.

\begin{tabular}{|c|c|c|c|c|c|c|c|c|c|c|c|}
\hline Species & $\mathrm{N}$ & Height (m) & $\begin{array}{l}\text { Crown } \\
\text { height } \\
(\mathrm{m})\end{array}$ & $\begin{array}{l}\text { Crown } \\
\text { width } \\
(\mathrm{Y} ; \mathrm{m})\end{array}$ & $\begin{array}{l}\text { Crown } \\
\text { width } \\
(\mathrm{X} ; \mathrm{m})\end{array}$ & $\begin{array}{l}\mathrm{CPH} \\
(\mathrm{m})\end{array}$ & $\begin{array}{l}\text { Tree mass } \\
(\mathrm{kg})\end{array}$ & $\begin{array}{l}\text { Diameter }^{\mathrm{z}} \\
(\mathrm{Y} ; \mathrm{mm})\end{array}$ & $\begin{array}{l}\text { Diameter }^{\mathrm{z}} \\
(\mathrm{X} ; \mathrm{mm})\end{array}$ & $\begin{array}{l}\text { Diameter }^{\mathrm{y}} \\
(\mathrm{Y} ; \mathrm{mm})\end{array}$ & $\begin{array}{l}\text { Diameter }^{\mathrm{y}} \\
(\mathrm{X} ; \mathrm{mm})\end{array}$ \\
\hline Freeman maple & 16 & $4.83(0.22)$ & $3.67(0.24)$ & $2.40(0.31)$ & $2.10(0.34)$ & $2.19(0.10)$ & $20.3(4.83)$ & $79.2(6.84)$ & $81.0(10.7)$ & $66.2(11.6)$ & $67.5(7.40)$ \\
\hline Swamp white oak & 13 & $4.45(0.19)$ & $3.46(0.23)$ & $3.23(0.58)$ & $3.20(0.53)$ & $1.76(0.07)$ & $22.1(4.75)$ & $88.7(8.67)$ & $90.2(8.93)$ & $76.3(8.28)$ & $76.5(7.05)$ \\
\hline Shingle oak & 18 & $4.62(0.46)$ & $3.57(0.46)$ & $4.04(0.57)$ & $3.79(0.63)$ & $1.99(0.22)$ & $25.0(8.04)$ & $89.9(9.55)$ & $90.4(10.5)$ & $73.9(10.3)$ & $75.4(9.61)$ \\
\hline
\end{tabular}

${ }^{\mathrm{z}}$ Measured $0.04 \mathrm{~m}(0.13 \mathrm{ft})$ above the root flare.

${ }^{\mathrm{y}}$ Measured $0.76 \mathrm{~m}(2.5 \mathrm{ft})$ above the root flare.

$\mathrm{CPH}=$ center of pressure height; $\mathrm{X}=$ direction perpendicular to the pickup truck's motion; $\mathrm{Y}=$ direction parallel to the truck's motion. 


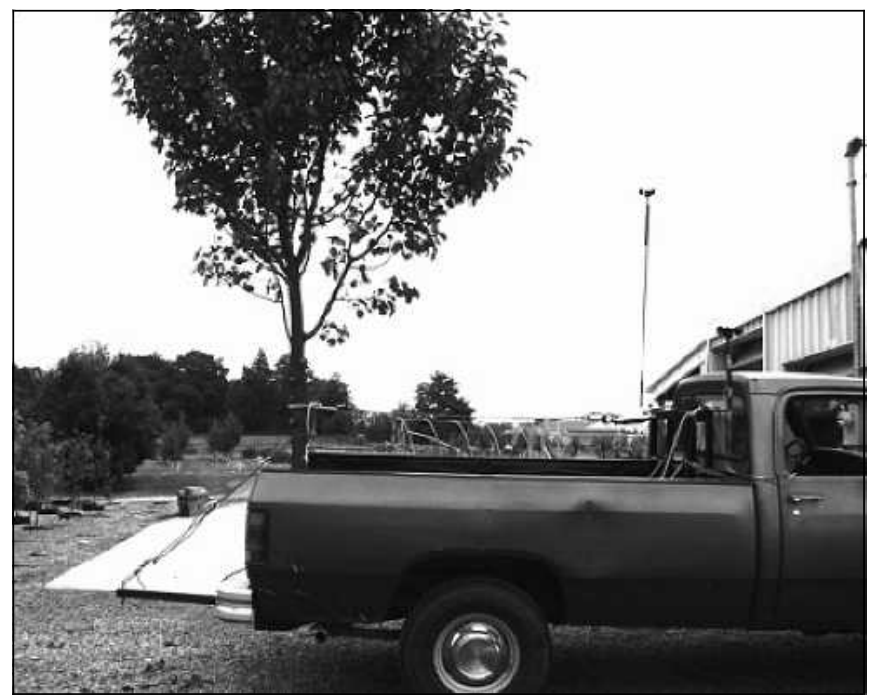

Figure 2. A tree secured to the sled in the bed of the pickup truck. The anemometers are visible in the front of the bed. The distance from the bottom to the top of the taller anemometer represents $2.4 \mathrm{~m}$ (7.9 ft).

that range to determine acceleration. Vehicular acceleration was multiplied by air density $\left(1.226 \mathrm{~kg} / \mathrm{m}^{3}\left[0.077 \mathrm{lb} / \mathrm{ft}^{3}\right]\right)$ and estimated crown volume to determine the force resulting from vehicular acceleration. Crown volume (V) was estimated using measured still-air crown dimensions (height $[\mathrm{h}]$ and width normal to vehicle direction [w]) and the formula for the volume of an egg (Narushin 2005), which visually approximated most crowns,

$$
\mathrm{V}=(0.6057-0.0018 \mathrm{w}) * \mathrm{hw}^{2}
$$

Because crowns are porous and reconfigure as wind speed increases, estimates of force resulting from acceleration were likely somewhat greater than the actual force. This bias may have varied among species as a result of differences in crown porosity and reconfiguration. The bias was small, however, because the force resulting from acceleration was less than $2 \%$ of drag at any wind speed. We subtracted the force resulting from vehicular acceleration from measured loads of each tree.

To use equation 2 to calculate drag from load measurements, it was necessary to determine the center of pressure height from a digital image of the crown. We removed any branches below the height of the cab of the truck and took high-resolution, stillair frontal images of tree crowns with an Olympus ${ }^{\circledR}$ Camedia digital camera, model C-2500L (Olympus America, Center Valley, PA). Images were segmented in Adobe ${ }^{\circledR}$ Photoshop ${ }^{\circledR}$ v. 6.0 (Adobe Systems, Inc., San Jose, CA) and scaled according to crown dimensions. Area and centroid of area were determined for each crown using ImageJ software (Wayne Rasband, Research Services Branch, National Institute of Mental Health, Bethesda, MD). The distance from the top of the truck's cab to the truck's bed $(0.97 \mathrm{~m}$ [3.2 ft]) was added to the centroid of crown area to determine the center of pressure height at which the resultant drag was assumed to act (see Figure 2 in Kane and Smiley [2006]). Because center of pressure height was determined from a still-air frontal image of each tree crown, deflection of distal twigs would lower the center of pressure height as wind speed increased. We did not account for this change directly, but estimated it by winching over red maples in a separate experiment. We winched trees over and measured trunk deflection per unit of trunk stress (see Kane et al. [in press] for a detailed description of this procedure). The estimated distal twig deflection would have increased drag values roughly 5\% (both oaks) and 10\% (Freeman maple). Presumably, twig deflection of reduction pruned trees would have been less because stiffness of distal twigs would increase after they were shortened.

Measured loads (L) (minus the force resulting from acceleration) were also converted into drag-induced bending moments (M) at the base of the tree,

$$
\mathrm{M}=\mathrm{L}^{*} 0.76
$$

where 0.76 is the distance from the base of the tree to the cable connecting the dynamometer to the tree. In addition to drag and bending moment, we measured tree mass, tree height, crown height, and crown width normal and parallel to wind direction before and after pruning.

\section{Pruning Treatments}

Trees received one of three pruning treatments: raising, reduction pruning, or thinning. Our intent was to mimic conditions faced by an arborist so all pruning was estimated visually by one person. For consistency, the same person pruned all trees in accordance with the ANSI A300 standard (ANSI 2001). For raised trees, all branches from the bottom $25 \%$ of the crown were removed. Maintaining crown shape, crown height and width, parallel and perpendicular to wind direction, were reduced by $25 \%$ for reduction-pruned trees. For thinned trees, approximately $25 \%$ of crown area was removed. For raising and reduction pruning, we chose $25 \%$ because it represented a meaningful but realistic change in crown height. For thinning, removal of $25 \%$ of crown area represented the maximum reduction in crown area suggested by the A300 Standard (ANSI 2001). Exact changes in crown area and tree mass were not known until images were analyzed and biomass removed was weighed, respectively. To account for variability in tree size before and after pruning, drag and bending moment were normalized by tree mass and crown area. Normalizing by mass, in particular, more accurately illustrated the effect of different pruning types because pruning types removed different amounts of mass and mass is an excellent predictor of drag and bending moment (Mayhead et al. 1975; Rudnicki et al. 2004; Vollsinger et al. 2005; Kane and Smiley 2006).

\section{Data Analysis}

We used an analysis of variance to investigate the effects of pruning and wind speed on drag, bending moment, drag per unit area, drag per unit mass, bending moment per unit area, and bending moment per unit mass. We used the least significant difference method and Tukey's honestly significant difference adjustment to separate means. We used regression analysis to determine which tree morphometric data best predicted postpruning drag and bending moment for each pruning type at 22.4 $\mathrm{m} / \mathrm{s}$ (50 mph). All analyses were performed using SAS v. 9 (SAS Institute, Cary, NC).

\section{RESULTS}

\section{Pruning Treatments}

Reduction of drag and bending moment differed by pruning type within each species (Table 2). For Freeman maple, reduction 
Table 2. Mean differences of drag $(\Delta \mathrm{D})$ bending moment ( $\Delta \mathrm{BM})$, drag per unit mass removed $(\Delta \mathrm{D} / \Delta \mathrm{m})$, bending moment per unit mass removed $(\Delta \mathrm{BM} / \Delta \mathrm{m})$, drag per unit area removed $(\Delta \mathrm{D} / \Delta \mathrm{A})$, and bending moment per unit area removed $(\Delta \mathrm{BM} / \Delta \mathrm{A})$ at $22.4 \mathrm{~m} / \mathrm{s}$ by pruning method. ${ }^{\mathrm{z}}$

\begin{tabular}{|c|c|c|c|c|c|c|c|}
\hline Species & Pruning method & $\Delta \mathrm{D}(\mathrm{N})$ & $\Delta \mathrm{BM}\left(\mathrm{N}^{*} \mathrm{~m}\right)$ & $\Delta \mathrm{D} / \Delta \mathrm{m}(\mathrm{N} / \mathrm{kg})$ & $\Delta \mathrm{BM} / \Delta \mathrm{m}\left(\mathrm{N}^{*} \mathrm{~m} / \mathrm{kg}\right)$ & $\Delta \mathrm{D} / \Delta \mathrm{A}\left(\mathrm{N} / \mathrm{m}^{2}\right)$ & $\Delta \mathrm{BM} / \Delta \mathrm{A}(\mathrm{N} / \mathrm{m})$ \\
\hline \multirow[t]{4}{*}{ Freeman maple } & Raise $(n=4)$ & $105(34.7) \mathrm{a}^{\mathrm{y}}$ & $214(168) \mathrm{a}$ & $1.75(0.96) \mathrm{a}$ & $1.00(5.10) \mathrm{a}$ & $-30.8(33.2) \mathrm{a}$ & $-127(119) \mathrm{a}$ \\
\hline & Thin $(\mathrm{n}=6)$ & 113 (46.7) a & $379(254) \mathrm{b}$ & $1.50(1.64) \mathrm{a}$ & $7.50(8.41) b$ & $-3.33(37.5) b$ & $12.8(85.6) b$ \\
\hline & Reduce $(\mathrm{n}=6)$ & $158(54.0) \mathrm{b}$ & $521(174) b$ & $3.50(1.87) b$ & $13.5(4.46) \mathrm{c}$ & $9.67(15.0) \mathrm{b}$ & $50.0(31.1) b$ \\
\hline & $P$ value & 0.0009 & $<0.0001$ & 0.006 & $<0.0001$ & $<0.0001$ & $<0.0001$ \\
\hline \multirow[t]{4}{*}{ Swamp white oak } & Raise $(\mathrm{n}=5)$ & $129(56.3) \mathrm{a}$ & 217 (134) a & $2.60(2.51) \mathrm{a}$ & $2.20(6.30) \mathrm{a}$ & $-60.6(41.9) a b$ & $-193(97.5) \mathrm{a}$ \\
\hline & Thin $(n=4)$ & $110(31.4) b$ & $224(88.8) \mathrm{a}$ & $2.50(1.00) \mathrm{a}$ & $3.50(2.65) \mathrm{a}$ & $-84.5(56.4) b$ & $-213(128) \mathrm{a}$ \\
\hline & Reduce $(n=4)$ & $85.5(49.6) b$ & $288(111) b$ & $0.50(2.38) \mathrm{b}$ & $6.00(5.29) \mathrm{b}$ & $-51.0(26.9) \mathrm{a}$ & $-92.0(56.7) b$ \\
\hline & $P$ value & 0.0001 & 0.0417 & 0.0001 & 0.0835 & 0.0259 & 0.0009 \\
\hline \multirow[t]{4}{*}{ Shingle oak } & Raise $(\mathrm{n}=7)$ & $174(103) \mathrm{a}$ & $296(164) \mathrm{a}$ & $1.43(3.31) \mathrm{a}$ & $-1.00(4.65) \mathrm{a}$ & $-112(47.8) a b$ & $-300(112) \mathrm{a}$ \\
\hline & Thin $(\mathrm{n}=5)$ & $107(79.1) \mathrm{a}$ & 236 (189) a & $0.20(3.27) \mathrm{a}$ & $0.80(6.50) a b$ & $-140(43.0) \mathrm{b}$ & $-334(109) \mathrm{a}$ \\
\hline & Reduce $(\mathrm{n}=6)$ & $109(63.1) \mathrm{a}$ & $277(160)$ a & $0.17(2.48) \mathrm{a}$ & $2.00(3.29) \mathrm{b}$ & $-91.3(48.1) \mathrm{a}$ & $-197(112) b$ \\
\hline & $P$ value & 0.0641 & 0.7196 & 0.7833 & 0.0143 & 0.0015 & $<0.0001$ \\
\hline
\end{tabular}

${ }^{\mathrm{z}}$ Standard deviations (in parentheses) follow each mean difference.

${ }^{\mathrm{y}}$ Means within a column followed by the same letter are not different by Tukey's honestly significant difference at $\alpha=0.05$.

pruning reduced drag more than thinning and raising, and raising was the least effective method for reducing bending moment. Reduction pruning and thinning reduced bending moment equally well. When reduction in drag and bending moment were normalized by mass removed during pruning, reduction pruning remained the most effective pruning type. Although raising and thinning were equally effective at reducing drag normalized by mass removed, thinning more effectively reduced bending moment per kilogram of mass removed during pruning than raising. Normalized by crown area removed during pruning, raising actually increased drag and bending moment per square meter of crown area, and thinning increased drag per square meter of crown area. Reduction pruning reduced both drag and bending moment more effectively than raising.

For swamp white oak, raising most effectively reduced drag, whereas reduction pruning most effectively reduced bending moment (Table 2). Raising and thinning were equally effective at reducing drag per kilogram of tree mass removed by pruning, whereas reduction pruning reduced bending moment per kilogram tree mass removed most effectively. Normalization by crown area removed caused all pruning types to increase drag and bending moment relative to the square meters of crown area removed; reduction pruning produced the smallest increase in drag and bending moment.

For shingle oak, there was some evidence that raising reduced drag more effectively than thinning or reduction pruning, but all treatments reduced bending moment equally well (Table 2). When drag and bending moment were normalized by tree mass removed, all pruning types reduced drag equally well, but reduction pruning reduced bending moment more effectively than raising. As occurred with swamp white oak, normalization by crown area removed caused all pruning types to increase drag

Table 3. Mean differences of drag $(\Delta D)$, bending moment $(\Delta B M)$, drag per unit mass removed $(\Delta D / \Delta m)$, bending moment per unit mass removed $(\Delta B M / \Delta m)$, drag per unit area removed $(\Delta D / \Delta A)$, and bending moment per unit area removed $(\Delta B M / \Delta A)$ by wind speed. ${ }^{2}$

\begin{tabular}{|c|c|c|c|c|c|c|c|}
\hline Species & $\begin{array}{l}\text { Wind speed } \\
(\mathrm{m} / \mathrm{s})\end{array}$ & $\Delta \mathrm{D}(\mathrm{N})$ & $\Delta \mathrm{BM}\left(\mathrm{N}^{*} \mathrm{~m}\right)$ & $\Delta \mathrm{D} / \Delta \mathrm{m}(\mathrm{N} / \mathrm{kg})$ & $\Delta \mathrm{BM} / \Delta \mathrm{m}\left(\mathrm{N}^{*} \mathrm{~m} / \mathrm{kg}\right)$ & $\Delta \mathrm{D} / \Delta \mathrm{A}\left(\mathrm{N} / \mathrm{m}^{2}\right)$ & $\Delta \mathrm{BM} / \Delta \mathrm{A}(\mathrm{N} / \mathrm{m})$ \\
\hline \multirow{6}{*}{$\begin{array}{l}\text { Freeman maple } \\
\qquad(\mathrm{n}=16)\end{array}$} & 22.4 & $128(5.62) \mathrm{a}^{\mathrm{y}}$ & 391 (16.6) a & $2.31(0.021) \mathrm{a}$ & $8.13(0.147) \mathrm{a}$ & $-5.31(1.05) \mathrm{a}$ & $-8.25(2.53) \mathrm{a}$ \\
\hline & 20.1 & $117(2.78) \mathrm{a}$ & $356(8.10) \mathrm{a}$ & $2.31(0.021) \mathrm{a}$ & $7.81(0.064) \mathrm{a}$ & $-3.31(0.529) \mathrm{a}$ & $-3.25(1.24) \mathrm{a}$ \\
\hline & 17.9 & $106(5.70) \mathrm{a}$ & $323(9.70) \mathrm{a}$ & $2.25(0.005) \mathrm{a}$ & $7.56(0.001) \mathrm{a}$ & $-1.25(0.003) \mathrm{a}$ & $1.31(0.059) \mathrm{a}$ \\
\hline & 15.6 & $95.4(2.79) \mathrm{a}$ & $288(8.39) \mathrm{a}$ & $2.13(0.026) \mathrm{a}$ & $7.38(0.046) \mathrm{a}$ & 0.75 (0.519) a & $6.50(1.28) \mathrm{a}$ \\
\hline & 13.4 & $84.7(5.56) \mathrm{a}$ & 255 (16.4) a & $2.13(0.026) \mathrm{a}$ & $6.94(0.160) \mathrm{a}$ & $2.81(1.05) \mathrm{a}$ & $11.4(2.55) \mathrm{a}$ \\
\hline & $P$ value & 0.0743 & 0.2848 & 0.9821 & 0.989 & 0.8479 & 0.8442 \\
\hline \multirow{6}{*}{$\begin{array}{l}\text { Swamp white oak } \\
\quad(\mathrm{n}=13)\end{array}$} & 22.4 & $110(2.60) \mathrm{a}$ & $241(4.16) \mathrm{a}$ & $1.92(0.156) \mathrm{a}$ & $3.76(0.335) \mathrm{a}$ & $-65.0(6.10) \mathrm{a}$ & $-168(15.5) \mathrm{a}$ \\
\hline & 20.1 & $107(1.73) \mathrm{a}$ & $233(1.85) \mathrm{a}$ & $2.38(0.023) \mathrm{a}$ & $4.38(0.156) \mathrm{a}$ & $-54.2(2.98) \mathrm{ab}$ & $-141(7.75) \mathrm{a}$ \\
\hline & 17.9 & $101(1.16) \mathrm{a}$ & 225 (4.61) a & $2.53(0.20) \mathrm{a}$ & $4.92(0.001) \mathrm{a}$ & $-43.9(0.006) \mathrm{ab}$ & $-114(0.040) \mathrm{ab}$ \\
\hline & 15.6 & $96.0(1.44) \mathrm{a}$ & $217(2.77) \mathrm{a}$ & $2.61(0.043) \mathrm{a}$ & $5.53(0.177) \mathrm{a}$ & $-33.3(3.05) \mathrm{ab}$ & $-86.8(7.89) a b$ \\
\hline & 13.4 & $91.0(2.89) \mathrm{a}$ & $217(2.77) \mathrm{a}$ & $2.84(0.110) \mathrm{a}$ & $6.00(0.312) \mathrm{a}$ & $-23.0(6.03) b$ & $-60.9(15.4) b$ \\
\hline & $P$ value & 0.7017 & 0.8233 & 0.7668 & 0.8097 & 0.0283 & 0.0116 \\
\hline \multirow{6}{*}{$\begin{array}{l}\text { Shingle oak } \\
\qquad(n=18)\end{array}$} & 22.4 & $134(4.86) \mathrm{a}$ & $273(10.8) \mathrm{a}$ & $0.67(0.092) \mathrm{a}$ & $0.50(0.183) \mathrm{a}$ & $-113(7.69) \mathrm{a}$ & $-276(17.9) \mathrm{a}$ \\
\hline & 20.1 & $124(2.44) \mathrm{a}$ & $250(5.19) \mathrm{a}$ & $0.94(0.027) \mathrm{a}$ & $0.94(0.076) \mathrm{a}$ & $-97.1(3.83) \mathrm{ab}$ & $-238(8.68) a b$ \\
\hline & 17.9 & $114(1.01) \mathrm{a}$ & $228(1.46) \mathrm{a}$ & $1.00(0.012) \mathrm{a}$ & $1.22(0.008) \mathrm{a}$ & $-81.4(0.024) a b c$ & $-203(0.194) a b c$ \\
\hline & 15.6 & $104(2.42) \mathrm{a}$ & $207(5.24) \mathrm{a}$ & $1.22(0.041) \mathrm{a}$ & $1.67(0.101) \mathrm{a}$ & $-65.3(3.88) b c$ & $-165(9.02) b c$ \\
\hline & 13.4 & $93.8(4.89) \mathrm{a}$ & 185 (10.6) a & $1.44(0.095) \mathrm{a}$ & $1.94(0.166) \mathrm{a}$ & $-49.7(7.66) \mathrm{c}$ & $-129(17.8) \mathrm{c}$ \\
\hline & $P$ value & 0.7208 & 0.7072 & 0.9369 & 0.9417 & $<0.0001$ & 0.0001 \\
\hline
\end{tabular}

${ }^{\mathrm{z}}$ Standard deviations (in parentheses) follow each mean difference.

${ }^{y}$ Means within a column followed by the same letter are not different by Tukey's honestly significant difference at $\alpha=0.05$. 


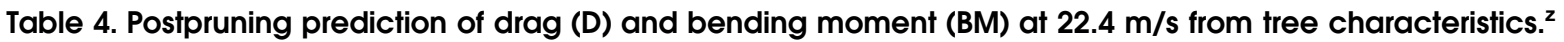

\begin{tabular}{|c|c|c|c|c|c|c|c|c|c|}
\hline Pruning type & & & $\begin{array}{l}\text { Tree mass } \\
(\mathrm{kg})\end{array}$ & $\begin{array}{l}\text { Tree height } \\
(\mathrm{m})\end{array}$ & $\begin{array}{l}\text { Crown height } \\
(\mathrm{m})\end{array}$ & $\begin{array}{l}\text { Crown width } \\
(\mathrm{m})^{\mathrm{y}}\end{array}$ & $\begin{array}{l}\text { Trunk diameter } \\
(\mathrm{m})^{\mathrm{x}}\end{array}$ & $\begin{array}{l}\text { Crown area } \\
\left(\mathrm{m}^{2}\right)\end{array}$ & $\begin{array}{l}\beta, \mathrm{B} \text { for best } \\
\text { predictor }\end{array}$ \\
\hline \multirow[t]{4}{*}{ Raise $(\mathrm{n}=16)$} & \multirow[t]{2}{*}{$\mathrm{D}$} & $P$ value & $<0.0001$ & 0.0187 & 0.0061 & $<0.0001$ & $<0.0001$ & 0.9804 & $28.7,36.6$ \\
\hline & & Adjusted $\mathrm{R}^{2}$ & 0.73 & 0.31 & 0.14 & 0.51 & 0.53 & -0.02 & \\
\hline & \multirow[t]{2}{*}{$\mathrm{BM}$} & $P$ value & $\overline{<0.0001}$ & 0.0015 & 0.0314 & 0.3183 & 0.0069 & 0.0906 & $53.3,53.6$ \\
\hline & & Adjusted $\mathrm{R}^{2}$ & 0.85 & 0.52 & 0.26 & 0.01 & 0.40 & 0.14 & \\
\hline \multirow[t]{4}{*}{ Reduce $(\mathrm{n}=16)$} & \multirow[t]{2}{*}{$\mathrm{D}$} & $P$ value & $\overline{<0.0001}$ & 0.6188 & 0.5786 & 0.0001 & 0.0009 & 0.8462 & $42.3,-26.0$ \\
\hline & & Adjusted $\mathrm{R}^{2}$ & 0.73 & -0.05 & -0.05 & 0.63 & 0.52 & -0.07 & \\
\hline & \multirow[t]{2}{*}{$\mathrm{BM}$} & $P$ value & $\overline{<0.0001}$ & 0.4705 & 0.0730 & 0.0044 & 0.0051 & 0.9131 & $118,-38.8$ \\
\hline & & Adjusted $\mathrm{R}^{2}$ & 0.81 & -0.03 & 0.16 & 0.41 & 0.40 & -0.07 & \\
\hline \multirow[t]{4}{*}{ Thin $(\mathrm{n}=16)$} & \multirow[t]{2}{*}{$\mathrm{D}$} & $P$ value & $\overline{<0.0001}$ & 0.0729 & 0.0517 & 0.0001 & 0.0001 & 0.9222 & $19.4,51.0$ \\
\hline & & Adjusted $\mathrm{R}^{2}$ & 0.72 & 0.16 & 0.19 & 0.52 & 0.61 & -0.07 & \\
\hline & \multirow[t]{2}{*}{$\mathrm{BM}$} & $P$ value & $\overline{<0.0001}$ & 0.0052 & 0.0026 & 0.0449 & 0.0051 & 0.4663 & $97.7,-10.1$ \\
\hline & & Adjusted $\mathrm{R}^{2}$ & 0.66 & 0.40 & 0.45 & 0.20 & 0.40 & -0.03 & \\
\hline
\end{tabular}

${ }^{\mathrm{z}}$ The best predictor (based on adjusted $\mathrm{R}^{2}$ value) is underlined and the slope $(\beta)$ and intercept (B) for that relationship are presented in the right-most column.

${ }^{\mathrm{y}}$ Calculated as the geometric mean of $\mathrm{X}$ and $\mathrm{Y}$ crown width.

${ }^{\mathrm{x}}$ Calculated as the geometric mean of $\mathrm{X}$ and $\mathrm{Y}$ trunk diameter at pivot point.

and bending moment, but the increase was smallest for reduction pruning.

\section{Wind Velocity}

Differences among pruning types were consistent at all wind speeds for each species. For all species, the absolute reduction in drag and bending moment resulting from pruning as well as the reduction in drag and bending moment per unit tree mass removed was consistent among wind speeds (Table 3). When normalized by crown area removed, however, drag and bending moment increased postpruning, and the increase was greater at greater wind speeds for shingle and swamp white oaks (Table 3).

\section{Drag and Tree Characteristics}

To a limited degree, pruning treatment influenced the tree characteristics (mass, height, crown height, crown width, crown area, and trunk diameter) that best predicted post-pruning drag (Table 4). With all species pooled together, tree mass was the best predictor of postpruning drag for all pruning treatments. Trunk diameter was the second best predictor of post-pruning drag for raised and thinned trees, whereas crown width was the second best predictor of post-pruning drag for reduced trees. For all pruning methods, crown area was the worst predictor of drag.
Across all pruning types, tree mass was the best predictor of post-pruning bending moment. Tree height was the second best predictor of post-pruning bending moment for raised trees, whereas crown width was the second best predictor of postpruning bending moments for reduced trees. Crown height was the second best predictor of post-pruning bending moments for thinned trees. Crown width was the worst predictor of bending moments for raised trees, whereas crown area was the worst predictor for reduced and thinned trees.

\section{Pruning Effects on Tree Dimensions}

The effect of pruning on tree dimensions (mass, center of pressure height, crown area, crown height, and crown width) differed by pruning treatment within each species (Table 5). For Freeman maple, reduction pruning reduced tree mass, center of pressure height, and crown width more than thinning and raising, whereas raising reduced crown height more than reduction pruning and thinning. Pruning treatments reduced crown area equally well. For swamp white oak, pruning treatments reduced tree mass and crown area equally well, but reduction pruning reduced the center of pressure height more than thinning and raising. Raising and reduction pruning reduced crown height more than thinning, and reduction pruning reduced crown width more than thinning.

Table 5. Mean percent change (percent $\Delta)^{z}$ in tree mass, center of pressure height (CPH), and crown area by treatment. ${ }^{y}$

\begin{tabular}{|c|c|c|c|c|c|c|}
\hline Species & Pruning method & Percent $\Delta$ tree mass & Percent $\Delta \mathrm{CPH}$ & Percent $\Delta$ crown area & Percent $\Delta$ crown height & Percent $\Delta$ crown width \\
\hline \multirow[t]{4}{*}{ Freeman maple } & Raise $(\mathrm{n}=5)$ & $11.7(1.92) \mathrm{a}^{\mathrm{x}}$ & $-8.50(3.38) \mathrm{a}$ & $28.9(1.72) \mathrm{a}$ & $24.2(6.00) \mathrm{a}$ & $6.61(1.31) \mathrm{a}$ \\
\hline & Thin $(\mathrm{n}=6)$ & $15.0(0.805) \mathrm{a}$ & $-2.35(0.273) \mathrm{a}$ & $20.7(2.13) \mathrm{a}$ & $0.00(0.00) \mathrm{b}$ & $0.00(0.00) \mathrm{a}$ \\
\hline & Reduce $(\mathrm{n}=6)$ & $23.7(3.09) \mathrm{b}$ & $5.61(3.29) \mathrm{b}$ & $26.8(0.599) \mathrm{a}$ & $12.4(0.894) \mathrm{c}$ & $21.1(5.31) \mathrm{b}$ \\
\hline & $P$ value & 0.0012 & 0.0038 & 0.6184 & $<0.0001$ & 0.0003 \\
\hline \multirow[t]{4}{*}{ Swamp white oak } & Raise $(\mathrm{n}=5)$ & $12.8(0.17) \mathrm{a}$ & $-10.6(2.00) \mathrm{a}$ & $48.0(0.735) \mathrm{a}$ & 13.4 (1.67) a & $7.83(0.307) \mathrm{a}$ \\
\hline & Thin $(\mathrm{n}=4)$ & $11.3(0.670) \mathrm{a}$ & $-3.29(1.92) \mathrm{a}$ & $51.2(2.70) \mathrm{a}$ & $0.00(0.00) \mathrm{b}$ & $0.00(0.00) \mathrm{b}$ \\
\hline & Reduce $(\mathrm{n}=4)$ & $13.3(0.485) \mathrm{a}$ & $7.28(8.02) b$ & $40.4(3.54) \mathrm{a}$ & $16.8(3.89) \mathrm{a}$ & $17.5(5.23) \mathrm{a}$ \\
\hline & $P$ value & 0.8136 & 0.0005 & 0.2311 & 0.0460 & 0.0126 \\
\hline \multirow[t]{4}{*}{ Shingle oak } & Raise $(\mathrm{n}=7)$ & $18.7(1.45) \mathrm{a}$ & $-8.91(3.17) \mathrm{a}$ & $57.8(1.54) \mathrm{a}$ & $8.15(0.585) \mathrm{a}$ & $0.00(0.00) \mathrm{a}$ \\
\hline & Thin $(\mathrm{n}=5)$ & $12.4(1.38) \mathrm{b}$ & $-1.30(0.08) \mathrm{a}$ & $57.8(1.89) \mathrm{a}$ & $0.00(0.00) \mathrm{b}$ & $0.00(0.00) \mathrm{a}$ \\
\hline & Reduce $(\mathrm{n}=6)$ & $14.4(0.206) \mathrm{b}$ & $4.50(2.52) \mathrm{b}$ & $46.5(3.37) \mathrm{a}$ & $20.6(4.93) \mathrm{c}$ & $21.9(6.53) b$ \\
\hline & $P$ value & 0.0025 & 0.0012 & 0.2523 & $<0.0001$ & $<0.0001$ \\
\hline
\end{tabular}

${ }^{\mathrm{z} C a l c u l a t e d:}$ [prepruning value - postpruning value]/[prepruning value].

${ }^{y}$ Standard deviations (in parentheses) follow each mean.

${ }^{\mathrm{x}}$ Means within a column followed by the same letter are not different by Tukey's honestly significant difference at $\alpha=0.05$. 
For shingle oak, raising removed more tree mass than reduction pruning and thinning, whereas reduction pruning reduced the center of pressure height, crown height, and crown width more than raising or thinning.

\section{DISCUSSION}

The difficulty in "eyeballing" pruning types was obvious because neither raising nor reduction pruning changed crown height in accordance with our intended 25\%, except for raising of Freeman maple. Because only entire branches were removed by raising, part of the disparity between intended and actual changes in crown height was the result of distal portions of remaining branches hanging below the height at which they were attached to the tree. Reduction of crown width was closer to the intended $25 \%$, and the consistently smaller percent change may reflect the pruner's conservative approach.

By itself, drag is a less useful measurement than bending moment because bending moment determines mechanical stress in the trunk. When trunk stress exceeds wood strength, the stem will break, so reduction in bending moment is critical when assessing tree risk. Because bending moment is the product of drag and center of pressure height, reducing either will reduce bending moment. Reducing the center of pressure height would be somewhat less effective because the percent change in drag was greater than the percent change in the center of pressure height. Reducing center of pressure height would be more effective for tall, narrow crowns because airflow may preferentially go around, as opposed to through, such crowns. This was evident for Freeman maples. For a tree with a short but dense crown, however, lowering the center of pressure would reduce bending moment less effectively. On larger trees, which have stiffer branches and would reconfigure less than small trees (Bertram 1989), drag may be proportional to the square of wind speed instead of the nearly linear relationship previously reported (Fraser 1962; Mayhead et al. 1975; Kane and Smiley 2006). In that case, reducing the center of pressure height would be less effective than reducing drag because of the linear relationship between center of pressure height and bending moment.

Differences among pruning types with respect to the reduction of drag and bending moment can be attributed to several factors with one caveat. To a limited degree, differences among pruning types regarding drag reduction were an artifact of the way drag was calculated. Because center of pressure height was used to convert measured loads into drag (equation 2), changes in center of pressure height would have affected post-pruning drag values even if measured loads had not changed. For example, raising increased the center of pressure height, which reduced drag, in part, because drag was inversely proportional to center of pressure height (equation 2). The reciprocal was true of reduction pruning, which lowered the center of pressure height for each species. This artifact best explained the paradoxic finding for both species of oak that differences among pruning types for drag reduction did not match differences among pruning types for the reduction of bending moment.

Considering that differences in drag reduction among pruning types were partially the result of changes in center of pressure height, and that, practically speaking, reduction in bending moment is more important than drag reduction, differences among pruning types are discussed chiefly with respect to reduction in bending moment.
Reduction in bending moment was attributable in large part to reduction in tree mass, regardless of pruning type, as reported previously (Smiley and Kane 2006). Because crown shape varied by species, the effect of pruning varied in accordance with pruning-induced changes in mass and shape. Thus, it was not surprising that reduction pruning and raising most effectively reduced bending moment for Freeman maple and shingle oak, respectively, because those pruning types removed the most mass for each respective species. Differences among pruning types with respect to reduction of bending moment per kilogram of mass removed, however, indicated that additional factors were involved. Pruning-induced changes in crown shape and fundamental mechanical principles (drag and bending moment) help explain the differences.

Reduction pruning was the most effective pruning treatment per unit mass removed for several reasons. Reduction pruning removed almost exclusively high drag elements from the crown because only distal (and therefore mostly foliated) portions of branches were removed. Leaves contribute more drag to tree crowns than branches and twigs (Vogel 1994), which was obvious when stripping red maples of foliage reduced drag and bending moment better than any pruning type (Smiley and Kane 2006). Reduction pruning also lowered the center of pressure height of the tree, further reducing the bending moment. Unlike the other pruning types, reduction pruning shortened branches, which behave as individual drag elements in the crown (Vollsinger et al. 2005). Shortening branches reduced both branch slenderness and the distance from the branch attachment to the centroid of area of the branch, at which the resultant drag on the branch can be assumed to act. Consequently, reduced branches would deflect less, increasing crown porosity as foliage reconfigured. Foliage reconfiguration differs among species (Vogel 1989), however, so testing of additional species is warranted. Lastly, reduction pruning reduced the depth of the crown, perhaps further facilitating airflow through the crown. The fact that crown width was the second best predictor of postpruning bending moment for reduction-pruned trees supported this notion.

After trees were raised, drag reduction was probably achieved by air passing more easily under the crown. It was unlikely that reduction in drag was the result of changes in crown porosity, because raising did not remove any branches from the interior of the crown. However, the greater postpruning center of pressure height presumably offset the reduction of drag and caused the reduction in bending moment to be comparatively small. The finding that tree height and not crown height was the second best predictor of postpruning bending moment for raised trees supported this idea because, although raising shortened crown length, it increased the center of pressure height. This was particularly evident for shingle oak, for which raising removed the most tree mass, but on a per mass removed basis, it was the least effective pruning method for reducing bending moment. Raising did not affect drag coefficient (Mayhead et al. 1975) but impeded crown reconfiguration (Fraser 1962) of a single Sitka spruce. In contrast with reduction pruning, raising removed a greater proportion of low drag elements from the crown because entire branches were removed. On a per mass removed basis, therefore, it was expected to be less effective at reducing bending moment. Crown reconfiguration was probably not affected by raising because individual branches were not modified like in reduction pruning. 
Of the three pruning types, thinning was the only one that did not change the shape of the crown. It was thus less likely that thinning would have facilitated airflow around the crown as may have been true for reduction pruning and raising. Vollsinger et al. (2005) observed that as wind speed increased, thinning did not noticeably change the pattern of crown area reduction (i.e., crown reconfiguration) of small deciduous trees in a wind tunnel. It thus makes sense that trunk diameter, a measure of tree size closely related to tree mass (Kane and Smiley 2006), was the second best predictor of postpruning bending moment for thinned trees as opposed to a measure of crown size as was true of reduction pruning and raising. The small increase in the center of pressure height did not appear to measurably offset the reduction in drag as a result of removal of foliage. This idea was supported by the fact that thinning was generally better at reducing bending moment per unit mass removed than raising. Thinned crowns were more porous, but branch deflection may have reduced porosity as wind speed increased. This would vary by species because wood stiffness would influence the amount of deflection. Individual branches may have experienced greater drag because they were not shortened and may have been more exposed after pruning (Vollsinger et al. 2005), which helps to explain why thinning was generally not as effective as reduction pruning in terms of reduction in bending moment per unit mass removed. This reasoning contradicts Mayhead et al.'s (1975) report of a smaller postpruning drag coefficient for Sitka spruce, but the disparity may be the result of the fact that the reported reduction in drag coefficient was probably primarily the result of a reduction in crown frontal area through reconfiguration as wind speed increased (Rudnicki et al. 2004; Vollsinger et al. 2005).

Although removing mass from the crown clearly reduces drag and bending moment, and this effect was most obvious when trees were stripped of all foliage (Smiley and Kane 2006), there are physiological limitations to this pruning approach. Removing too much foliage reduces photosynthesis and may cause undue physiological stress (Ennos 1997). Furthermore, removing foliage and twigs exclusively from the proximal portions of branches may reduce branch taper, which can increase the likelihood of failure (Cremer et al. 1982; Putz et al. 1983; Petty and Swain 1985). Staebler (1963) demonstrated a reduction in trunk taper when more than two-thirds of the lower branches were removed. Thus, arborists need to be able to maximize the reduction in bending moment while maintaining an appropriate amount of photosynthetic material in the crown. In this regard, reduction pruning was better than raising and, to a lesser extent, thinning.

It was not entirely clear why crown area was such a poor predictor of post-pruning drag and bending moment, because previous studies have shown it to be a reasonably reliable predictor of drag and bending moment of unpruned trees (Kane and Smiley 2006; Kane et al. in press). Crown area's inability to predict post-pruning drag and bending moment was presumably related to the post-pruning increase in drag and bending moment per unit crown area for most pruning types and species. For all pruning types, the post-pruning percent reduction in crown area appeared to be unrealistically high for both species of oak. This was consistent with the prevalence of negative values for postpruning reduction in drag and bending moment per square meter of crown area removed for those species. Inspection of the crown images confirmed that $50 \%$ crown area reduction was unrealis- tic, so image analysis difficulties probably played a role. Compressing a three-dimensional crown into two dimensions may have clouded the analysis. Leeward branches may have been hidden by windward foliage in still air photographs, which would underestimate the actual surface area to which drag is related (equation 1). The likelihood of hiding leeward twigs would have been greater in wider crowns, and both oaks had wider crowns than Freeman maple. The results may not be entirely attributed to image analysis error, however, because Vollsinger et al. (2005) also observed an increase in post-pruning drag after removing one-third of branch mass on small deciduous trees. Crowns of their trees were small and appeared to be quite sparse, so image analysis was less likely to have been an issue.

\section{CONCLUSIONS}

Reduction of drag and bending moment were strongly correlated with the mass of foliage and twigs removed, whereas the effect of wind speed was essentially constant for the range of wind speeds tested. Future studies should attempt to investigate the effect of pruning at greater wind speeds, which are more likely to cause structurally sound trees to fail. Species must also be considered when selecting a pruning type because crown shape will influence how much biomass pruning removes. Wood properties and the ability of foliage to reconfigure, which influence a crown's porosity after pruning, are also related to species. A limitation of this and all other pruning studies to date is that comparatively small trees were tested. This is especially true with respect to the logarithmic increase in wind speed with height above the ground (Davenport 1968). The anemometers attached to the truck in the current study did not measure a change in wind speed between the $1.4 \mathrm{~m}(4.6 \mathrm{ft})$ that separated them, but for larger trees, the wind speed at the top of the crown may be substantially greater than at the base. Theoretically, raising would even less effectively reduce drag than reduction pruning assuming such an increase in wind speed with height. Testing small trees is also problematic because they deflect and reconfigure more effectively than large trees. Although no trunks or branches failed during testing, it was not possible to determine whether this reflected the greater flexibility of small trees, the lack of structural defects (e.g., decay or weak branch attachments) on trees, or a combination of these factors. Trees were also exposed to a static force during testing, which does not reflect the gusty conditions that occur in reality. Prediction of tree failure based on static forces overestimates the wind speed at which failures have been documented (Oliver and Mayhead 1974).

Pruning recommendations cannot be developed exclusively in light of mechanical considerations. Physiological considerations are also important as are the incidence of decay and regrowth after pruning. Further studies are needed to help determine pruning recommendations in terms of species, age, health, site conditions, tree risk, and aesthetics. The need to investigate dose is less important because much work, including the current study, supports the linear relationship between mass reduction and drag or bending moment reduction (Fraser 1962; Mayhead et al. 1975; Rudnicki et al. 2004; Vollsinger et al. 2005; Smiley and Kane 2006).

Acknowledgments. This study was funded in part by a USDA FS grant through the National Urban and Community Forestry Advisory Council (NUCFAC). We acknowledge John Homyk, John James, and Nathan 
White for helping to collect data, the Bartlett Tree Research Lab for use of the sled for testing, and two anonymous reviewers for helpful comments on earlier drafts of this manuscript.

\section{LITERATURE CITED}

American National Standards Institute (ANSI A300 part 1). 2001. American National Standard for Tree Care Operations-Tree, Shrub and Other Wood Plant Maintenance-Standard Practices (Pruning). Tree Care Industry Association, Manchester, NH.

Bertram, J.E.A. 1989. Size-dependent differential scaling in branches: The mechanical design of trees revisited. Trees (Berlin) 4:241-253.

Cremer, K.W., C.J. Borough, F.H. McKinnell, and P.R. Carter. 1982. Effects of stocking and thinning on wind damage in plantations. New Zealand Journal of Forestry Science 12:244-268.

D'Amato, N.E., T.D. Sydnor, and D.K. Struve. 2002. Urban foresters identify Ohio's tree needs. Journal of Arboriculture 28:291-301.

Davenport, A.G. 1968. Wind Effects on Buildings and Structures. Univ. Toronto Press Toronto, Ontario, Canada.

Dirr, M.A. 1997. Dirr's Hardy Trees and Shrubs. Timber Press, Portland, OR. 493 pp.

Ennos, AR. 1997. Wind as an ecological factor. Trends in Ecology and Evolution 12:108-111.

Fraser, A.I. 1962. Wind Tunnel Studies of the Forces Acting on the Crowns of Small Trees. Rep. Forest. Res. HMSO, U.K. pp. 178-183.

Kane, B., M. Pavlis, J.R. Seiler, and J.R. Harris. 2008. Crown reconfiguration and trunk stress in deciduous trees. Canadian Journal of Forest Research (in press).

Kane, B., and E.T. Smiley. 2006. Drag coefficients and crown area estimation of red maple. Canadian Journal of Forest Research 36: 1951-1958.

Lilly, S.J. 2001. Arborists' Certification Study Guide. International Society of Arboriculture, Champaign, IL. 222 pp.

Mayhead, G.J., J.B.H. Gardiner, and D.W. Durrant. 1975. A report on the physical properties of conifers in relation to plantation stability. Unpublished report. Forestry Commission Research and Development Division, Roslin, Midlothian, UK.

Narushin, V.G. 2005. Egg geometry calculation using the measurements of length and breadth. Poultry Science 84:482-484.

Oliver, H.R., and G.J. Mayhead. 1974. Wind measurements in a pine forest during a destructive gale. Forestry 47:185-194.

Petty, J.A., and C. Swain. 1985. Factors influencing stem breakage of conifers in high winds. Forestry. 58:75-84.

Putz, F.E., P.D. Coley, K. Lu, A. Montalvo, and A. Aiello. 1983. Uprooting and snapping of trees: Structural determinants and ecological consequences. Canadian Journal of Forest Research 13:1011-1020.

Rudnicki, M., S.J. Mitchell, and M.D. Novak. 2004. Wind tunnel measurements of crown streamlining and drag relationships for three conifer species. Canadian Journal of Forest Research 34:666-676.

Smiley, E.T., and B. Kane. 2006. The effects of pruning type on wind loading of Acer rubrum. Arboriculture and Urban Forestry 32:33-40.

Staebler, G.R. 1963. Growth along the stems of full-crowned Douglasfir trees after pruning to specified heights. Journal of Forestry 61: 124-127.

Vogel, S. 1989. Drag and reconfiguration of broad leaves in high wind. Journal of Experimental Botany 78:941-948.

1994. Life in Moving Fluids. 2nd Ed. Princeton University Press, Princeton, NJ.

Vollsinger, S., S.J. Mitchell, K.E. Byrne, M.D. Novak, and M. Rudnicki. 2005. Wind tunnel measurements of crown streamlining and drag relationships for several hardwood species. Canadian Journal of Forest Research 35:1238-1249.

Wessolly, L. 1995. Fracture diagnosis of trees part 2: statics-Integrated methods-Statically-integrated assessment (SIA) the practitioner's method of diagnosis. Stadt und Gruen 8:570-573.

\author{
Michael Pavlis \\ Virginia Tech University \\ Department of Forestry \\ Blacksburg, VA, U.S.
}

Brian Kane (corresponding author)

University of Massachusetts

Department of NRC

126 Holdsworth Hall

Amherst, MA 01003, U.S.

bkane@nrc.umass.edu

\section{J. Roger Harris \\ Virginia Tech University \\ Department of Horticulture \\ Blacksburg, VA, U.S.}

John R. Seiler

Virginia Tech University

Department of Forestry

Blacksburg, VA, U.S.

Résumé. L'élagage peut permettre de diminuer les risques de bris en réduisant le moment de flexion induit lors de la poussée, mais il y a peu d'études qui quantifient cet effet. Nous avons simulé un vent en tirant sur des arbres à l'aide d'une camionnette à des vitesses de 0 à 22,4 $\mathrm{m} / \mathrm{sec}$. et avons par la suite mesuré le moment de flexion qui était induit tout comme des données morphométriques de l'arbre, et ce sur des érables Freeman (Acer $\times$ freemanii), des chênes bicolores (Quercus bicolor Willd.) et des chênes imbriqués (Quercus imbricaria Michx.). Toutes les mesures ont été prises avant et après chacun que des trois types d'élagage (regaussement de couronne, réduction de cime et éclaircissage) définis dans la norme américaine ANSI A300 aient été effectués. La diminution du moment de flexion induit différait selon le type d'élagage et selon les espèces, et ce en accord avec la masse de feuillage et de branches retirées. L'efficacité des types d'élagage était de ce fait reliée à l'espèce, et ce du fait que l'architecture affectait la quantité de masse de cime élaguée selon chaque type d'élagage. En général, pour chaque unité de masse de cime retirée, la réduction de cime produisait une diminution accrue du moment de flexion induit, et ce contrairement à l'éclaircissage ou le rehaussement de couronne. Ceci était dû à une diminution de la hauteur du centre de pression et probablement à un accroissement de la porosité de la cime suite à l'élagage. La prédiction de la diminution du moment de flexion induit n'était pas fiable si on se basait sur la surface de la couronne après l'élagage. Nous discutons d'applications pratiques de nos recherches.

Zusammenfassung. Rückschnitt kann das Risiko des Baumversagens durch die Reduzierung des Biegemoments vermindern, aber es gibt wenige Studien, die diesen Effekt quantifizieren. Wir simulierten Windlast, indem wir Bäume in Pickup-Trucks bei einer Geschwindigkeit von 0 $22,4 \mathrm{~m} / \mathrm{s}$ durch die Gegend fuhren und maßen den Windlasteintrag über das Biegemoment und die morphometrischen Daten für folgende Bäume: (Acer $\times$ freemanii), (Quercus bicolor Willd.), und (Quercus imbricaria Michx.). Alle Messungen wurden vor und nach einer von drei ANSI A300 Schnittmaßnahmen durchgeführt. Die Reduktion des widerstandsabhängigen Biegemoments variierte bei den unterschiedlichen Schnittmaßnahmen, hauptsächlich durch die Menge an entfernten Blättern und Zweigen. Die Effektivität der Schnitttypen wurde daher mit der Art in Beziehung gesetzt, da die arttypische Kronenarchitektur die Menge des entfernten Materials beeinflusste. Allgemein reduzierte die Maßnahme: Kroneneinkürzung dieses Biegemoment mehr als die Maßnahmen: Aufasten und Ausdünnen. Das lag an der Herabsetzung des Druckzentrums und wahrscheinlich an der Kronenporosität nach dem Rückschnitt. Eine Vorhersage der Reduktion des Biegemoments 
anhand der reduierten Kroenfläche nach dem Rückschnitt war nicht verlässlich. Wir diskutieren hier die praktischen Anwendungen unserer Ergebnisse.

Resumen. La poda puede disminuir el riesgo de fractura del árbol al reducir el momento de doblamiento debido al efecto de vela, pero hay pocos estudios que cuantifiquen este efecto. Se simuló el viento llevando los árboles en una camioneta a 0 a $22.4 \mathrm{~m} / \mathrm{s}(0$ a $55 \mathrm{mph})$ para el medir el momento de doblamiento como también los datos morfomètricos del árbol, para maple Freeman (Acer $\times$ freemanii), encino blanco de los pantanos (Quercus bicolor Willd.), y encino (Quercus imbricaria Michx.). Todas las mediciones fueron tomadas antes y después de que fueran aplicados cada uno de los tres tipos de poda ANSI (elevación, reducción, aclareo). La disminución del momento de doblamiento difirió según el tipo de poda dentro de cada especie, de acuerdo a la masa de follaje y ramas removidas. La efectividad de los tipos de poda estuvo por tanto relacionada por la especie, siendo que la arquitectura de la copa afectó qué tanta masa por cada tipo de poda fue removida. En general, por unidad de masa removida, la poda de reducción fue más efectiva que las otras dos. Esto se debió a que se bajó la altura del centro de presión y, presumiblemente, al incremento de la porosidad de de la copa después de la poda. La predicción de la reducción del momento por el efecto de vela no fue confiable con base en la reducción en el área de la copa después de la poda. Se discuten las aplicaciones prácticas de estos hallazgos. 